\title{
Isothermal Melt Crystallization Kinetics Study of Biodegradable Poly(3-hydroxybutyrate)/Multiwalled Carbon Nanotubes Nanocomposites
}

\author{
By Changling XU and Zhaobin QIU*
}

KEY WORDS: Biodegradable Polymer / Carbon Nanotubes / Nanocomposites / Isothermal Crystallization /

Poly(3-hydroxybutyrate) (PHB) is a truly biodegradable and highly biocompatible polymer; ${ }^{1-6}$ however, wider application range of $\mathrm{PHB}$ has been limited because of its stiffness and brittleness problems. Moreover, its melting temperature is so close to the thermal decomposition temperature that $\mathrm{PHB}$ becomes thermally unstable during processing. Therefore, many attempts have been made to improve its mechanical and thermal properties, among which polymer blending is an easy and convenient way of achieving desirable combination of properties. Ha et al. and Di Lorenzo et al. have recently reviewed miscibility, properties and biodegradability of polymer blends containing PHB. ${ }^{7,8}$

Addition of nano-particles such as carbon nanotubes (CNTs) to form nanocomposites has provided another means to improve materials performances. ${ }^{9,10}$ CNTs possess unique mechanical, electrical and thermal properties that make it ideal candidates to substitute conventional nano-fillers in the fabrication of polymer nanocomposites. ${ }^{1-16}$ It is noted that the strong interfacial adhesion between polymer chains and nanotubes is critically important to effectively maximize load transfer from the matrix to CNTs. Recently, PHB/single walled carbon nanotubes (SWCNTs) nanocomposites have been prepared by solution casting method using chloroform as cosolvent. ${ }^{17}$ The experimental results showed that the crystalline size substantially decreased for the PHB/SWCNTs nanocomposite with a $1 \%$ weight fraction of SWCNTs as compared to that of neat PHB; moreover, the polymer nanocomposite films showed an increase in hardness and Young's modulus with increasing SWCNTs contents. ${ }^{17}$

To the best of our knowledge, biodegradable PHB/multiwalled carbon nanotubes (MWNTs) nanocomposites have not been reported so far in the literature. We have recently prepared PHB/MWNTs nanocomposites at different MWNTs loadings by solution casting method and studied the effects of the presence of MWNTs and their loadings on the subsequent multiple melting behavior of PHB in the nanocomposites crystallized non-isothermally from the melt. ${ }^{18}$ In this note, we further studied the isothermal melt crystallization kinetics of neat $\mathrm{PHB}$ and the $\mathrm{PHB} / \mathrm{MWNT}$ nanocomposites for a better understanding of the influence of the presence of MWNTs and the loadings on the crystallization behavior of PHB in the PHB/MWNTs nanocomposites.

\section{EXPERIMENTAL}

\section{Materials and Preparation of PHB/f-MWNTs Nanocom- posites}

Natural PHB $\left(M_{\mathrm{w}} \approx 2.0 \times 10^{5}\right)$ was kindly supplied by Biomer Company, Germany. The received PHB was purified by dissolving in chloroform and precipitating in excess of methanol. The obtained sample was further died in a vacuum oven for $7 \mathrm{~d}$ at $50^{\circ} \mathrm{C}$. Acid treated multiwalled carbon nanotubes containing carboxylic groups (f-MWNTs) were purchased from Chengdu Institute of Organic Chemistry, Chinese Academy of Sciences. The f-MWNTs are approximately $30-50 \mathrm{~nm}$ in diameter and are ranging between 10 and $20 \mu \mathrm{m}$ in length.

PHB/f-MWNTs nanocomposites were prepared using chloroform as the mutual solvent. The f-MWNTs loadings are 0.5 and $1 \mathrm{wt} \%$ in the PHB/f-MWNTs nanocomposites. The typical preparation procedure is as follows. The appropriate amount of f-MWNTs was added into the chloroform. Then, the mixture was sonicated with a KQ $3200 \mathrm{E}$ ultrasonic generator at $150 \mathrm{~W}$ to make a uniformly dispersed suspension. Meanwhile, PHB was placed into chloroform at elevated temperature and stirred for $1 \mathrm{~h}$ to dissolve PHB completely. Next, the PHB solution was added to the f-MWNTs suspension, and sonication was continued, with stirring for $6 \mathrm{~h}$. The PHB/ f-MWNTs solution was poured into a dish to evaporate the solvent at room temperature for about $24 \mathrm{~h}$. The sample was further dried at $70^{\circ} \mathrm{C}$ under vacuum for $72 \mathrm{~h}$ to remove the solvent completely. In this work, neat PHB and its two PHB/ f-MWNTs nanocomposites were abbreviated as 100/0, 99.5/ 0.5 and $99 / 1$ respectively, with the first number referring to PHB while the second number referring to f-MWNTs.

\section{Characterizations}

A field emission scanning electron microscopy (S-4700, 
Hitachi Co.) was used to observe the morphology of the surfaces of $\mathrm{PHB} / \mathrm{f}-\mathrm{MWNT}$ s nanocomposites, which were fractured in liquid nitrogen. All specimens were coated with gold before examination. A Hitachi H-800 Transmission electron microscopy (TEM) was also used to investigate the dispersion of f-MWNTs in the PHB matrix. Thin sections (with thickness of about $50-70 \mathrm{~nm}$ ) of the nanocomposites was performed under cryogenic conditions $\left(-80^{\circ} \mathrm{C}\right)$ using a Leica EM FC6 ultramicrotome.

Thermal analysis was performed using a TA Instruments differential scanning calorimetry (DSC) Q100 with a Universal Analysis 2000. Samples weights were in the range of 4-6 mg. Two different procedures were employed:

Nonisothermal Melt Crystallization. The samples were first heated to $190^{\circ} \mathrm{C}$ at $40^{\circ} \mathrm{C} / \mathrm{min}$ (first heating), held at $190^{\circ} \mathrm{C}$ for $3 \mathrm{~min}$ to erase any thermal history, cooled to $20^{\circ} \mathrm{C}$ at $20^{\circ} \mathrm{C} /$ min (first cooling), and heated again to $190^{\circ} \mathrm{C}$ at $20^{\circ} \mathrm{C} / \mathrm{min}$ (second heating). The crystallization peak temperature was obtained from the first cooling traces.

Isothermal Melt Crystallization. The samples were heated to $190^{\circ} \mathrm{C}$ at $40^{\circ} \mathrm{C} / \mathrm{min}$, held for $3 \mathrm{~min}$ to erase any thermal history and then cooled to the chosen crystallization temperature at $60^{\circ} \mathrm{C} / \mathrm{min}$. The samples were held at the chosen crystallization temperature for a period of time until the crystallization was complete. The crystallization temperatures chosen in this work were from 115 to $135^{\circ} \mathrm{C}$.

Wide angle X-ray diffraction (WAXD) experiments were performed on a Rigaku D/Max $2500 \mathrm{VB} 2 \mathrm{t} / \mathrm{PC}$ X-ray diffractometer at $40 \mathrm{kV}$ and $200 \mathrm{~mA}$ at room temperature in the range of $5-40^{\circ}$ with a scanning rate of $4^{\circ} / \mathrm{min}$. The samples were pressed into films with a thickness of around $0.5 \mathrm{~mm}$ on a hot stage at $190^{\circ} \mathrm{C}$ and then transferred into a vacuum oven at $120^{\circ} \mathrm{C}$ for $3 \mathrm{~d}$.

\section{RESULTS AND DISCUSSION}

\section{Dispersion of f-MWNTs in the PHB Matrix}

It is well known that a homogeneous dispersion of $\mathrm{f}-$ MWNTs and strong interfacial interactions between the polymer matrix and carbon nanotubes can effectively improve the mechanical, electrical and thermal performances of the polymer matrix because they are the key factors of influencing the physical properties and their end use of polymer nanocomposites. ${ }^{11-18}$ Therefore, the dispersion of f-MWNTs in the PHB matrix was studied first in this work.

Figure 1a shows the SEM image of a 99/1 nanocomposite. It can be seen from Figure 1a that f-MWNTs are homogeneously dispersed throughout the polymer matrix and no apparent aggregation is found. The bright regions in the SEM image were attributed to f-MWNTs due to their high electrical conductivity. An interesting phenomenon of f-MWNTs, with two ends strongly embedded in the matrix, demonstrates a strong interfacial adhesion between f-MWNTs and the PHB matrix. Figure $1 \mathrm{~b}$ shows a typical TEM image of the ultrathin section of the 99/1 nanocomposite. As shown in Figure 1b, individual f-MWNTs are randomly dispersed within the

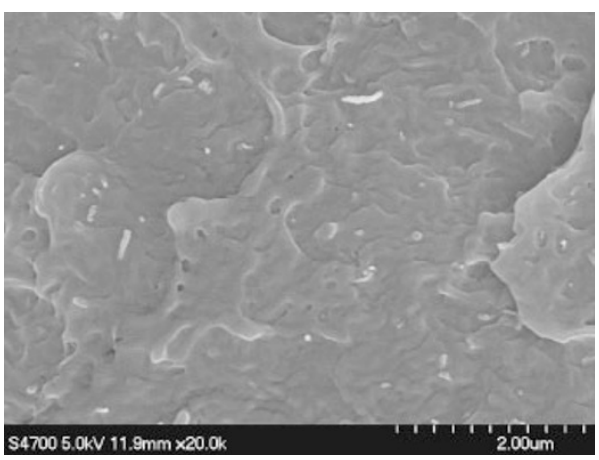

(a)

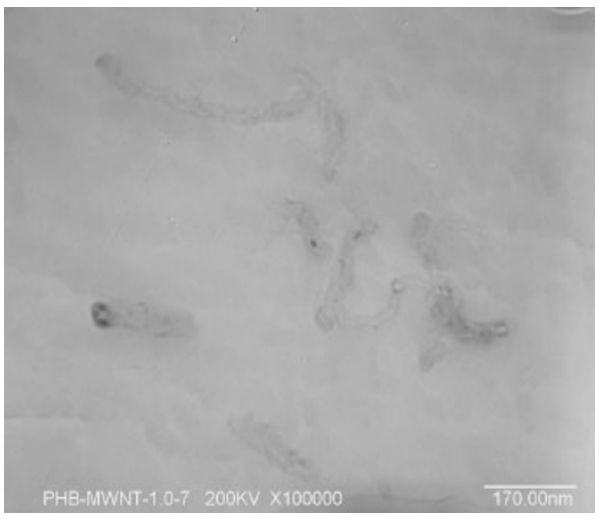

(b)

Figure 1. (a) SEM image of a 99/1 nanocomposite, (b) TEM image of a 99/1 nanocomposite from the ultrathin section.

polymer matrix without preferred alignment or orientation and no apparent f-MWNTs aggregation is observed. Furthermore, most f-MWNTs remain curved in the nanocomposite, due to an extreme flexibility of nanotubes. Both SEM and TEM observations suggest a homogeneous distribution of f-MWNTs throughout the PHB matrix.

\section{Isothermal Melt Crystallization Kinetics of Neat PHB and Its Nanocomposites}

It is of great interest to study the effect of the addition of f-MWNTs on the crystallization behavior of PHB in the nanocomposites. The effect of f-MWNTs on the nonisothermal melt crystallization of PHB was studied first with DSC at a cooling rate of $20^{\circ} \mathrm{C} / \mathrm{min}$. For brevity, the related plots are not shown here. As a result, neat PHB has a crystallization peak temperature $\left(T_{\mathrm{p}}\right)$ at around $93.3^{\circ} \mathrm{C}$, which shifts to high temperature range in the presence of f-MWNTs. In the case of $99.5 / 0.5$ and $99 / 1$ samples, $T_{\mathrm{p}} \mathrm{s}$ shift to around 97.3 and $98.2^{\circ} \mathrm{C}$, respectively, indicating that the presence of f-MWNTs enhances the crystallization of PHB in the nanocomposites compared to neat PHB. In general, homogeneous nucleation starts spontaneously from the melt and requires more time, whereas heterogeneous nucleation forms as soon as the samples reach the crystallization temperature. ${ }^{19,20}$ The fact that f-MWNTs are well dispersed in the PHB matrix leads to an 


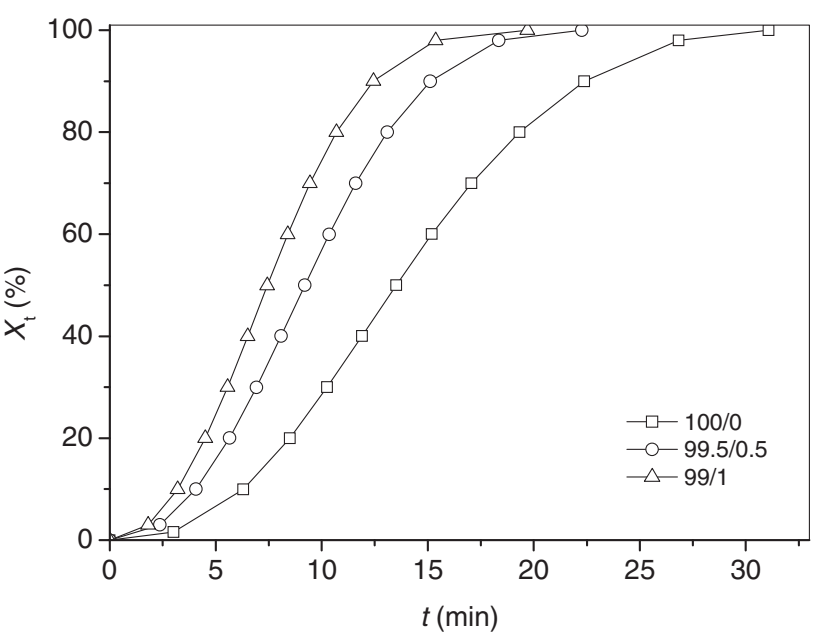

(a)

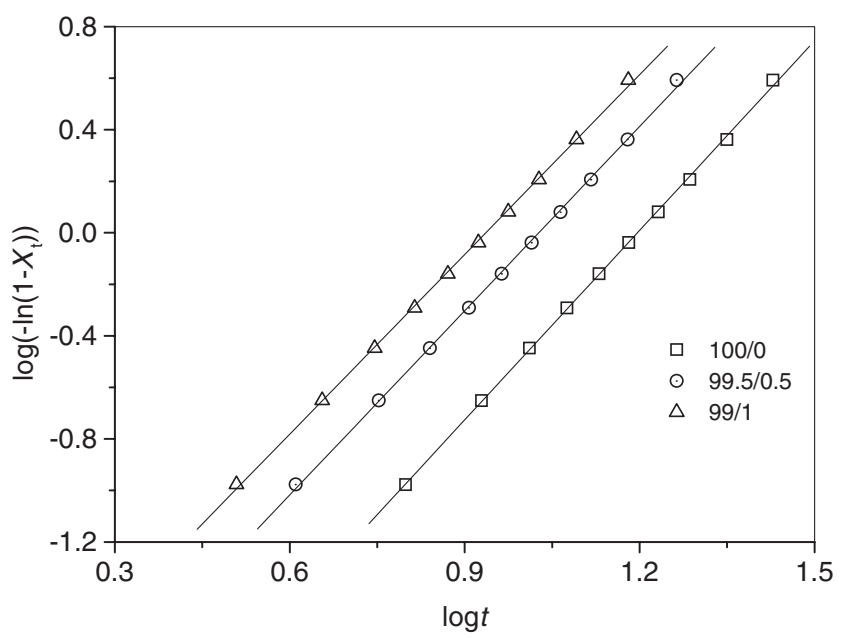

(b)

Figure 2. (a) Development of relative crystallinity with crystallization time for neat $\mathrm{PHB}$ and its nanocomposites crystallized isothermally at $125^{\circ} \mathrm{C}$, (b) the related Avrami plots of neat PHB and its nanocomposites.

increased number of sites available for the nucleation of PHB spherulites, thereby facilitating the crystallization process.

Isothermal melt crystallization kinetics of neat PHB and its two nanocomposites were further studied in a wide range of crystallization temperatures $\left(T_{\mathrm{c}} \mathrm{s}\right)$. The effect of the f-MWNTs loadings on the isothermal melt crystallization of PHB was studied first. Figure 2a illustrates the development of relative crystallinity with crystallization time for both neat PHB and the $\mathrm{PHB} / \mathrm{f}-\mathrm{MWNT}$ nanocomposites at $125^{\circ} \mathrm{C}$, respectively. It can be seen that all these curves have the similar sigmoid shape. Furthermore, the corresponding crystallization time for the PHB/f-MWNTs nanocomposites becomes shorter with increasing the f-MWNTs loadings. For example, it took neat PHB almost $32 \mathrm{~min}$ to complete crystallization at $125^{\circ} \mathrm{C}$; however, for the $99.5 / 0.5$ and $99 / 1$ nanocomposites, the total crystallization time was only around 22 and $19 \mathrm{~min}$, respectively. It is clear that the addition of f-MWNTs accelerated
Table I. Isothermal melt crystallization kinetic parameters for neat $\mathrm{PHB}$ and the PHB/f-MWNTs nanocomposites based on the Avrami equation

\begin{tabular}{cccc}
\hline Samples & $\begin{array}{c}T_{\mathrm{c}} \\
\left({ }^{\circ} \mathrm{C}\right)\end{array}$ & $n$ & $\begin{array}{c}k \\
\left(\mathrm{~min}^{-\mathrm{n}}\right)\end{array}$ \\
\hline $100 / 0$ & 110 & 2.78 & $4.36 \times 10^{-1}$ \\
& 115 & 2.63 & $2.34 \times 10^{-2}$ \\
& 120 & 2.50 & $4.17 \times 10^{-3}$ \\
& 125 & 2.45 & $1.17 \times 10^{-3}$ \\
& 130 & 2.27 & $1.66 \times 10^{-4}$ \\
$99.5 / 0.5$ & 115 & 2.40 & $1.17 \times 10^{-1}$ \\
& 120 & 2.37 & $2.57 \times 10^{-2}$ \\
& 125 & 2.35 & $3.89 \times 10^{-3}$ \\
& 130 & 2.34 & $5.01 \times 10^{-4}$ \\
& 135 & 2.17 & $1.11 \times 10^{-4}$ \\
& 115 & & \\
& 120 & 2.44 & $1.73 \times 10^{-1}$ \\
& 125 & 2.28 & $3.31 \times 10^{-2}$ \\
& 130 & 2.37 & $7.24 \times 10^{-3}$ \\
& 135 & 2.37 & $1.26 \times 10^{-3}$ \\
& & 2.50 & $1.02 \times 10^{-4}$ \\
\hline
\end{tabular}

the isothermal crystallization of PHB in the PHB/f-MWNTs nanocomposites compared with neat $\mathrm{PHB}$.

The well-known Avrami equation is used to analyze the overall isothermal crystallization kinetics of both neat PHB and its nanocomposites. It assumes that the relative degree of crystallinity $X_{\mathrm{t}}$ develops as a function of crystallization time $t$ as follows: ${ }^{21,22}$

$$
1-X_{\mathrm{t}}=\exp \left(-k t^{\mathrm{n}}\right)
$$

Where $X_{\mathrm{t}}$ is the relative crystallinity at time $t, k$ is the crystallization rate constant and $n$ is the Avrami exponent depending on the nature of nucleation and growth geometry of the crystals. Figure $2 \mathrm{~b}$ shows the Avrami plots for neat PHB and its two nanocomposites at $125^{\circ} \mathrm{C}$, from which the Avrami parameters $n$ and $k$ are obtained from the slopes and intercepts of the Avrami plots, respectively. In addition, the influence of $T_{\mathrm{c}}$ on the isothermal melt crystallization for neat PHB and its nanocomposites was also investigated. For brevity, the related plots are not shown here.

For comparison, Table I summarizes the related crystallization kinetics parameters for the overall isothermal crystallization of neat PHB and its nanocomposites at various $T_{\mathrm{c}} \mathrm{s}$. As shown in Table I, the values of $n$ are between 2 and 3 for both neat PHB and its nanocomposites, indicating that the crystallization of PHB might correspond to the spherulitic growth with heterogeneous nucleation. ${ }^{23}$ Since the unit of $k$ is $\min ^{-n}$ and $n$ is not constant, it is difficult to compare the crystallization rate directly from the values of $k$. Therefore, another parameter, the crystallization half-time $t_{1 / 2}$, was introduced in order to describe crystallization kinetics.

$$
t_{1 / 2}=\left(\frac{\ln 2}{k}\right)^{1 / n}
$$

$t_{1 / 2}$ is defined as the time taken from the onset of the crystallization until $50 \%$ completion of the crystallization 


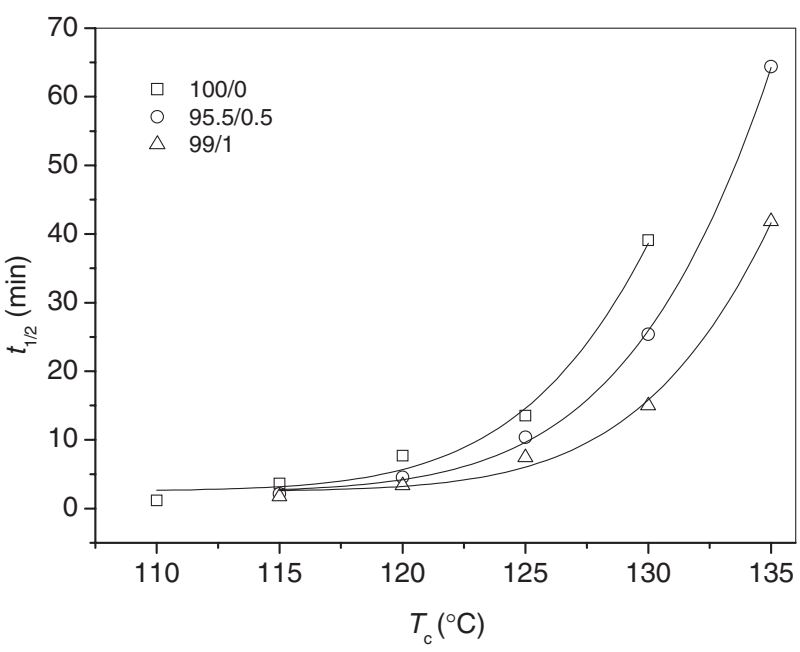

(a)

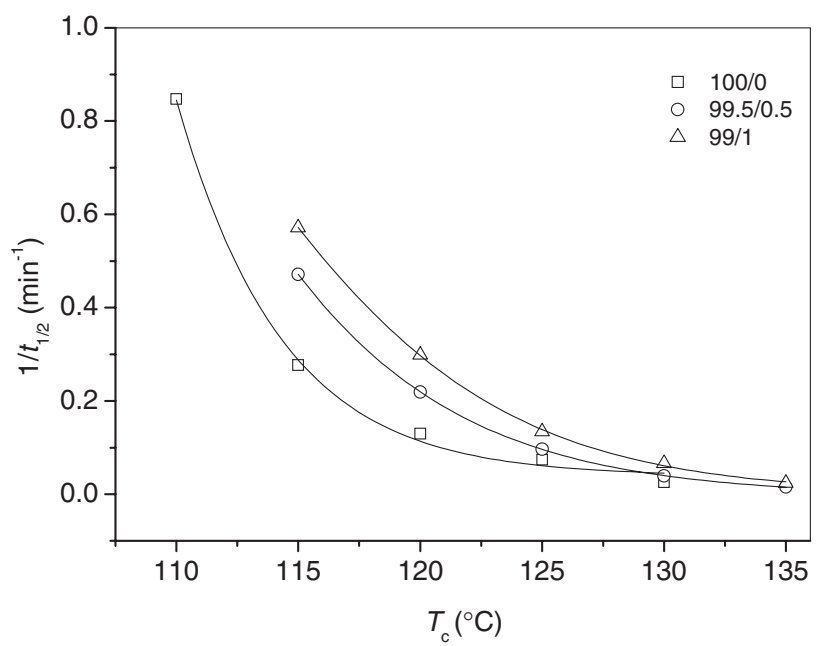

(b)

Figure 3. Temperature dependence of $t_{1 / 2}$ (a) and $1 / t_{1 / 2}$ (b) for neat PHB and its nanocomposites.

process. The crystallization rate can also be easily described by the reciprocal of $t_{1 / 2}$.

Parts a and $\mathrm{b}$ of Figure 3 illustrate the variation of $t_{1 / 2}$ and $1 / t_{1 / 2}$ with $T_{\mathrm{c}}$ for neat PHB and its two nanocomposites, respectively, from which the effects of $T_{\mathrm{c}}$ and f-MWNTs loadings on the variation of overall crystallization rate can be obtained clearly. As shown in Figure $3 \mathrm{a}$, the values of $t_{1 / 2}$ increase with increasing $T_{\mathrm{c}}$ for both neat PHB and its two nanocomposites with different f-MWNTs loadings, indicating that the crystallization is retarded at higher $T_{\mathrm{c}}$. For a given $T_{\mathrm{c}}$, the values of $t_{1 / 2}$ decrease with increasing the f-MWNTs loadings in the PHB/f-MWNTs nanocomposites as compared with those of neat $\mathrm{PHB}$, suggesting that the addition of f-MWNTs enhances the crystallization of PHB in the PHB/ f-MWNTs nanocomposites. In addition, Figure $3 \mathrm{~b}$ shows the variation of $1 / t_{1 / 2}$ with $T_{\mathrm{c}}$ for both neat PHB and its two nanocomposites at different f-MWNTs loadings, from which we can obtain the similar conclusions that the overall

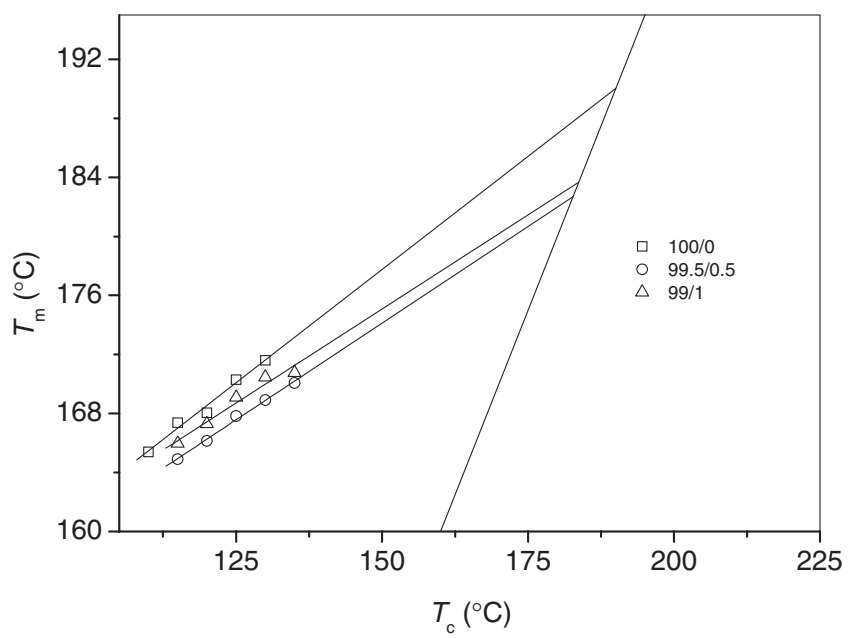

Figure 4. Hoffman-Weeks plots of neat PHB and its nanocomposites for the estimation of the equilibrium melting points.

crystallization rates of PHB are enhanced with decreasing $T_{\mathrm{c}}$ and increasing the f-MWNTs loadings in the PHB/f-MWNTs nanocomposites.

The equilibrium melting points of neat $\mathrm{PHB}$ and its nanocomposites at different f-MWNTs loadings were further studied since it may provide some information on the miscibility between PHB and f-MWNTs. ${ }^{24}$ The equilibrium melting points can be determined with the Hoffman-Weeks equation: ${ }^{25}$

$$
T_{\mathrm{m}}=\eta T_{\mathrm{c}}+(1-\eta) T_{\mathrm{m}}{ }^{\mathrm{o}}
$$

Where $\eta$ is a stability parameter, $T_{\mathrm{m}}$ is the apparent melting temperature, and $T_{\mathrm{m}}{ }^{\mathrm{o}}$ is the equilibrium melting point. Figure 4 illustrates the Hoffman-Weeks plots, from which $T_{\mathrm{m}}{ }^{\mathrm{o}}$ of neat PHB is estimated to be around $190.0^{\circ} \mathrm{C}$. In the case of $99.5 / 0.5$ and 99/1 samples, the $T_{\mathrm{m}}{ }^{\circ}$ values shift to around 182.7 and $183.7^{\circ} \mathrm{C}$, respectively. The depression of $T_{\mathrm{m}}{ }^{\circ}$ for PHB in the nanocomposites indicates that there is some limited miscibility between PHB and f-MWNTs because of functional group of $\mathrm{COOH}^{24}$

WAXD experiments were performed to investigate the effect of the addition of f-MWNTs on the crystal structure of PHB. Figure 5 shows the WAXD patterns of neat PHB and its nanocomposites with different f-MWNTs loadings. Neat PHB presents three main diffraction peaks at around $13.4^{\circ}, 16.8^{\circ}$ and $22.7^{\circ}$ which can be assigned to (020), (110), and (111) planes of PHB, respectively. ${ }^{7}$ The nanocomposites also exhibit nearly the same diffraction peaks at the same locations, indicating that incorporating with the f-MWNTs does not modify the crystal structure of PHB. However, it should be noted that the diffraction peaks shift to around $13.2^{\circ}$ and $16.6^{\circ}$ for the $99 / 1$ sample. Such slight shift to lower angle suggests that the corresponding interplanar spacings increase with the addition of f-MWNTs. Consequently, the unit cell parameters and the crystal volume are slightly higher in the 99/1 nanocomposite than in neat $\mathrm{PHB}$, indicating that the presence of $1 \mathrm{wt} \% \mathrm{f}$ MWNTs suppresses the compact packing of the PHB chains 


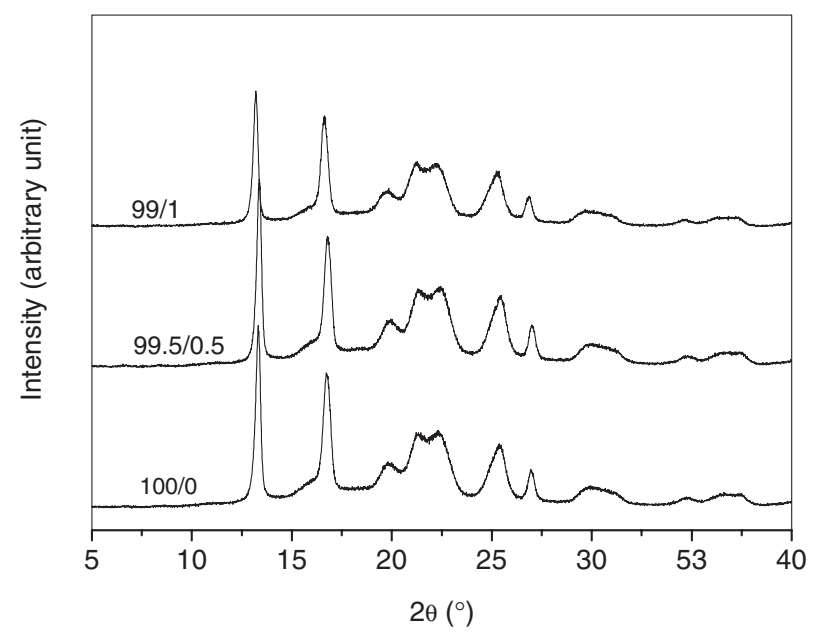

Figure 5. WAXD patterns of neat PHB and its nanocomposites.

during crystallization. Similar results were also found in the poly(L-lactide)/MWNTs nanocomposites. ${ }^{26}$ From Figure 5, it can also be found that the corresponding diffraction peaks intensity values are almost the same for neat PHB and the 99.5/ 0.5 nanocomposite and slightly higher than those for the $99 / 1$ nanocomposite. Thus, the values of degree of crystallinity are estimated to be around $72 \%$ for neat PHB and the 99.5/0.5 nanocomposite; however, the degree of crystallinity is reduced to be around $65 \%$ for the $99 / 1$ nanocomposite.

\section{CONCLUSIONS}

PHB/f-MWNTs nanocomposites have been prepared successfully through solution blending method in this work. Both SEM and TEM observations indicate a fine and homogeneous dispersion of f-MWNTs throughout the PHB matrix. The effect of $\mathrm{f}-\mathrm{MWNT}$ s on the isothermal melt crystallization kinetics of PHB was investigated by DSC in detail. The overall crystallization rates are faster in the PHB/f-MWNTs nanocomposites than in neat PHB at a given $T_{\mathrm{c}}$; furthermore, the overall crystallization rates decrease with increasing $T_{\mathrm{c}}$ for both neat PHB and the PHB/f-MWNTs nanocomposites. The acceleration of isothermal crystallization process of PHB in the PHB/ f-MWNTs nanocomposites is attributed to the heterogeneous nucleation effect of f-MWNTs. Equilibrium melting points of PHB/f-MWNTs nanocomposites are lower than that of neat PHB, suggesting that there may be some limited miscibility between PHB and f-MWNTs arising from their interaction. It is also found that the addition of f-MWNTs does not modify the crystal structure of $\mathrm{PHB}$ in the $\mathrm{PHB} / \mathrm{f}-\mathrm{MWNT}$ nanocomposites.
Acknowledgment. The authors thank Biomer, Germany for kindly providing PHB samples. Part of this work is financially supported by the National Natural Science Foundation, China (Grant No. 20774013), Program for New Century Excellent Talents in University (NCET-06-0101) and the project of Polymer Chemistry and Physics, Beijing Municipal Commission of Education (XK100100640).

Received: May 10, 2009

Accepted: June 24, 2009

Published: August 12, 2009

\section{REFERENCES}

1. Y. Doi, "Microbial Polyesters," VCH Publishers, New York, 1990.

2. G. A. Van der walle, G. J. De koning, R. A. Weuthuis, and G. Egggink, Adv. Biochem. Eng./Biotechnol., 71, 263 (2001).

3. M. Vert, Biomacromolecules, 6, 538 (2005).

4. C. Bastioli, "Handbook of Biodegradable Polymers," Smithers Rapra Press, UK, 2005.

5. E. Chiellini and R. Solaro, "Recent Advances in Biodegradable Polymers and Plastics," Wiley-VCH, Weinheim, 2003.

6. R. A. J. Verlinden, D. J. Hill, M. A. Kenward, C. D. Williams, and I. Radecka, J. Appl. Microbiol., 102, 1437 (2007).

7. C. S. Ha and W. J. Cho, Prog. Polym. Sci., 27, 759 (2002).

8. M. Di Lorenzo, M. Raimo, E. Cascone, and E. Martusceli, J. Macromol. Sci., Part B: Phys., 40, 639 (2001).

9. S. Iijima, Nature, 354, 56 (1991).

10. M. Moniruzzaman and K. I. Winey, Macromolecules, 39, 5194 (2006).

11. T. X. Liu, I. Y. Phang, L. Shen, S. Y. Chow, and W. D. Zhang, Macromolecules, 37, 7214 (2004).

12. W. D. Zhang, L. Shen, I. Y. Phang, and T. X. Liu, Macromolecules, 37, 256 (2004).

13. T. Kashiwagi, E. Grulke, J. Hilding, R. Harris, W. Awad, and J. Douglas, Macromol. Rapid Commun., 23, 761 (2002).

14. S. Y. Yang, J. R. Castilleja, E. V. Barrera, and K. Lozano, Polym. Degrad. Stab., 83, 383 (2004).

15. J. Yang, Y. H. Lin, J. F. Wang, M. F. Lai, J. Li, J. J. Liu, X. Tong, and H. M. Cheng, J. Appl. Polym. Sci., 98, 1087 (2005).

16. G. X. Chen, H. S. Kim, B. H. Park, and J. S. Yoon, Polymer, 47, 4760 (2006).

17. S. L. Yun, E. Gerry, G. E. Gadd, B. A. Latella, V. Lo, R. A. Russell, and P. J. Holden, Polym. Bull., 61, 267 (2008).

18. C. L. Xu and Z. B. Qiu, J. Polym. Sci., Part B: Polym. Phys., submitted.

19. J. Y. Kim, H. S. Park, and S. H. Kim, Polymer, 47, 1379 (2006).

20. S. H. Kim, S. H. Ahn, and T. Hairai, Polymer, 44, 5625 (2003).

21. M. Avrami, J. Chem. Phys., 7, 1103 (1939).

22. M. Avrami, J. Chem. Phys., 9, 177 (1941).

23. B. Wunderlich, "Macromolecular Physics," Academic Press, New York, 1976 vol. 2.

24. T. M. Wu and E. C. Chen, J. Polym. Sci., Part B: Polym. Phys., 44, 598 (2006).

25. J. D. Hoffman and J. J. Weeks, J. Chem. Phys., 42, 4301 (1965).

26. Y. Zhao, Z. Qiu, and W. Yang, J. Phys. Chem. B, 112, 16461 (2008). 